Revista General de Información y Documentación ISSN: 1132-1873

http://dx.doi.org/10.5209/RGID.60807

\title{
Análisis bibliométrico comparativo de la actividad científica del CSIC y cuatro homólogos europeos: CNRS, HG, MPG Y CNR (2006-2015)
}

\author{
Jairo Maldonado Carrillo후 ${ }^{1}$ Michela Montesi ${ }^{2}$
}

Recibido: 16 de enero 2018 / Aceptado: 4 de junio de 2018

Resumen. Se describe la actividad investigadora del Consejo Superior de Investigaciones Científicas (CSIC) en la década 2006-1015 comparativamente con cuatro instituciones europeas similares: el Centre National de la Recherche Scientifique (CNRS), el Helmholtz-Gemeinschaft (HG), el MaxPlanck-Gesellschaft (MPG), y el Consiglio Nazionale delle Ricerche (CNR). Los datos relativos a actividad, impacto y colaboración se obtuvieron de Scopus. Concretamente se estudiaron, entre los indicadores de actividad, el número de publicaciones y el índice de actividad; entre los indicadores de impacto, el número de citas y citas por publicación, el porcentaje de publicaciones no citadas, el índice h y el índice de inmediatez; y, entre los indicadores de colaboración, la frecuencia de colaboración internacional y el índice de colaboración internacional. Los resultados ponen de manifiesto diferencias entre las instituciones estudiadas en el volumen de la producción, en el impacto alcanzado, y en la especialización. EL CSIC se configura como la institución que consigue la mejor relación presupuesto-producción-impacto. Las demás instituciones destacan en aspectos diferentes, tales como el volumen de la producción realizada (CNRS), el impacto alcanzado (MPG), el grado de especialización (HG), o la escasa actividad de colaboración internacional (CNR).

Palabras clave: Indicadores bibliométricos; Consejo Superior de Investigaciones Científicas (CSIC); Centre National de la Recherche Scientifique (CNRS); Helmholtz-Gemeinschaft (HG); Max-Planck-Gesellschaft (MPG); Consiglio Nazionale delle Ricerche (CNR); evaluación científica; análisis institucional.

\section{[en] Comparative bibliometric analysis of the scientific activity of the CSIC and four European counterparts: CNRS, HG, MPG and CNR (2006-2015)}

\begin{abstract}
The article presents the research activity of the Spanish Consejo Superior de Investigaciones Científica (CSIC) during the period 2006-2015 in comparison with similar European institutions, i.e. the Centre National de la Recherche Scientifique (CNRS), the HelmholtzGemeinschaft (HG), the Max-Planck-Gesellschaft (MPG), y the Consiglio Nazionale delle Ricerche (CNR). Using data from Scopus, production, impact and collaboration are analyzed. Specifically, the following indicators are produced: number of publications and activity index, for the research activity indicators; number of citations and citations per publication, percentage of no cited items, h-index and immediacy index, for the impact indicators; and, finally, frequency of international collaboration and international collaboration index, for the collaboration indicators. Results show differences among the

$1 \quad$ Universidad Complutense de Madrid

E-mail: jairomal@ucm.es

2 Universidad Complutense de Madrid. Departamento de Biblioteconomía y Documentación

E-mail: mmontesi@ucm.es
\end{abstract}


five research centers in all the three dimensions studied. The CSIC appears to be the institution that achieves the best budget-production-impact balance. The other institutions all stand out in different aspects, such as total production (CNRS), impact (MPG), degree of specialization (HG), or limited international collaboration (CNR).

Keywords: Bibliometric indicators; Consejo Superior de Investigaciones Científicas (CSIC); Centre National de la Recherche Scientifique (CNRS); Helmholtz-Gemeinschaft (HG); MaxPlanck-Gesellschaft (MPG); Consiglio Nazionale delle Ricerche (CNR); research evaluation; institutional analysis.

Sumario. 1. Introducción. 2. Instituciones objeto de comparación. 3. Metodología. 4. Resultados. 5. Conclusiones. 6. Referencias bibliográficas.

Cómo citar: Maldonado Carrillo, J., Montesi, M. (2018) Análisis bibliométrico comparativo de la actividad científica del CSIC y cuatro homólogos europeos: CNRS, HG, MPG Y CNR (2006-2015), en Revista General de Información y Documentación 28 (1), 163-191.

El presente artículo es una versión revisada, modificada y abreviada de un Trabajo de Fin de Máster defendido en septiembre de 2017 en la Universidad Complutense de Madrid y disponible en formato digital en la dirección web: http://eprints.ucm.es/45176/

\section{Introducción}

El objetivo del presente artículo es el de medir, analizar y evaluar la producción científica del Consejo Superior de Investigación científicas (CSIC) en la década 2006-2015 a partir de datos de Scopus, para compararla con la de otros centros de investigación europeos similares, concretamente el francés Centre National de la Recherche Scientifique (CNRS), los alemanes Helmholtz-Gemeinschaft (HG) y Max-Planck-Gesellschaft (MPG), y el italiano Consiglio Nazionale delle Ricerche (CNR). Los cinco centros analizados en este estudio representan las primeras cinco instituciones científicas gubernamentales de Europa occidental en el SCImago Institutions Rankings o SIR de 2015 (SCImago Lab, 2015).

La actividad científica global del Consejo Superior de Investigación Científicas se analiza anualmente a través de informes que el propio CSIC elabora a efectos de evaluación, siendo Bordons y otros (2016) el último publicado para el periodo 2011-2015. De forma complementaria, existen numerosos trabajos que estudian diferentes aspectos de la actividad del CSIC, centrándose en una o varias áreas de investigación en concreto, examinando la producción científica en ciertas ramas, como Bordons y Costas (2005), en el que se analiza la producción del CSIC en el área de recursos naturales, o explorando ciertos fenómenos de la comunicación científica en el contexto del CSIC. Entre los varios aspectos de la comunicación científica estudiados, cabe mencionar los patrones de colaboración, el orden de la autoría, o la distribución por género de la plantilla de la institución. Ortega (2011), por ejemplo, intenta esclarecer qué clase de relaciones de colaboración establecen varios grupos de investigación del CSIC con diferentes socios locales, nacionales e internacionales, mientras que De Filippo y otros (2008) examina la parte de la producción del CSIC que es fruto de la colaboración del centro español con el 
mundo científico latinoamericano. Costas y Bordons (2011) analizan los patrones de autoría en un conjunto de trabajos publicados por el personal de investigación del CSIC en las áreas de Recursos Naturales, Ciencia de los Materiales, y Biología y Biomedicina, destacando la influencia de la edad y categoría académica en el orden de los autores. En cuestiones de género, Mauleón y otros (2008) evalúa por separado la producción de los investigadores y las investigadoras del CSIC de las áreas de biología y biomedicina, mientras que Ortega (2017) analiza la presencia masculina y femenina de la plantilla entera del CSIC en diferentes redes sociales de corte académico, como Academia.edu, ResearchGate y Google Scholar Citations. Finalmente, muchos trabajos se centran en el análisis de trayectorias de investigación individuales, como Ayala-Gascón y otros (2012), en donde se analiza la producción personal de un destacado científico del CSIC, o Costas y otros (2010), en donde se pone a prueba una nueva metodología específicamente pensada para llevar a cabo análisis bibliométricos a nivel individual.

Por otro lado, en cuanto a los trabajos en los que se compare la producción científica del CSIC con la de otros centros de investigación internacionales similares, la bibliografía disponible es limitada. De entre los pocos estudios recuperados se pueden destacar el de Sánchez (1999), en el que se compara la actividad científica del CSIC con la del conjunto de España y Europa, y el de Albert y otros (2007), en el que se compara la producción del CSIC con la del CNRS francés y el CNR italiano en el área de biotecnología. Son más numerosos los estudios disponibles en los que se compara la producción del CSIC con la de otras instituciones nacionales dedicadas a la investigación, como universidades u hospitales. De entre éstos, sólo los ya mencionados informes de autoevaluación que realiza anualmente el propio CSIC, en los que compara su actividad con la del conjunto de universidades del país, analizan la producción al completo. El resto se centra en determinadas áreas de investigación, como es el caso del estudio de López-Muñoz y otros (1996), en el que se examina la producción científica española en el ámbito de la neurociencia.

También cabe señalar que la mayor parte de estos trabajos previos utilizan la base de datos Web of Science (WoS) como fuente de datos, sobre todo aquellos cuyas temáticas de estudio están relacionadas con alguna rama de las ciencias naturales o algún campo de la tecnología, áreas de conocimiento mejor representada en esta base de datos. En el caso de las investigaciones que analizan la producción de algún campo de las ciencias sociales o de las humanidades, se suele recurrir a otro tipo de bases de datos de alcance preferentemente nacional, como, por ejemplo, ISOC, Teseo o Dialnet. Sin embargo, ninguna de las publicaciones que emplean alguna de estas bases de datos trata en particular la producción del CSIC, sino que suelen analizar el conjunto de la producción española para un campo de investigación determinado, como, por ejemplo, en el artículo de Arguimbau-Vivó y otros (2013), en el que se estudia la producción científica española del área de la cienciometría recogida en ISOC. Lo mismo ocurre con los pocos estudios encontrados que utilizan Scopus como fuente: no estudian la producción del CSIC específicamente sino un ámbito de conocimiento particular, 
como en el caso del trabajo de Romo-Fernández y otros (2013), en donde se analiza la producción científica española en materia de energías renovables.

El uso de Scopus como fuente de datos para un análisis bibliométrico de la producción del CSIC puede aportar cierta ventaja frente WoS, que según algunos podría considerarse un subconjunto perfecto de Scopus (Waltman, 2016). Hasta la aparición en noviembre de 2004 de Scopus y Google Académico (o Google Scholar), WoS era la única herramienta disponible para el análisis y la evaluación de la producción científica (Orduña-Malea y otros, 2016). El contenido de Scopus y WoS se encuentra sesgado por idioma, campos de investigación y procedencia geográfica. En Scopus, por ejemplo, ciertas especialidades, como la biomedicina, las ciencias naturales o la ingeniería, se pueden ver favorecidas en detrimento de áreas como las ciencias sociales o las humanidades, aunque la mayor representación de las humanidades en WoS se da solo para revistas en inglés (Bosman y otros, 2006). Lo mismo ocurre con el idioma: ambas bases de datos dan clara prioridad a trabajos procedentes de revistas en lengua inglesa, aunque esto parece menos acentuado en el caso de Scopus, que es bastante más inclusivo en términos de revistas indizadas recopilando aproximadamente el doble de revistas que WoS (Mongeon y Paul-Hus, 2016; Thelwall y Kousha, 2015). A estos sesgos debidos en gran parte a las políticas no siempre transparentes de selección e inclusión de revistas, debe añadir una larga lista de errores, que en el caso de Scopus, algunos han llegado a definir "horrores" (Franceschini y otros, 2016), como, por ejemplo, la remoción de las citas obtenidas por las versiones Online First de los artículos, la asignación del mismo DOI a artículos diferentes, o las citas "fantasmas" que se adscriben injustificadamente a ciertos artículos. Otro problema detectado en la base de datos Scopus sería la duplicación de artículos que se atribuyen por error a diferentes revistas (Valderrama-Zurián y otros, 2015). A pesar de todas estas limitaciones y aunque sería recomendable ir explotando nuevas fuentes de datos bibliométricos, como por ejemplo los disponibles gracias a la infraestructura del acceso abierto (Waltman, 2016), en la actualidad la gran mayoría de los estudios bibliométricos siguen tomando como fuente de datos estas dos herramientas.

Con estas premisas, el presente trabajo pretende complementar la literatura previa existente, mostrando una comparativa de la producción global del CSIC con la de instituciones europeas similares en lugar que con instituciones nacionales (universidades y hospitales), por un lado, y basándose en datos procedentes de Scopus, en lugar que WoS, por otro, aún compartiendo con los estudios anteriores el enfoque metodológico. Cuando se compara la producción del CSIC con la de universidades y hospitales españoles, se incurre en un sesgo de partida, pues mientras que el personal de éste se dedica en exclusiva a la investigación, el personal universitario y hospitalario debe compaginarla con la docencia o la práctica clínica, y sus resultados en investigación se ven condicionados por esta menor dedicación, por lo menos en términos de producción. Con lo cual, de alguna manera, estamos redimensionando la actividad investigadora del CSIC realizando una comparación “entre pares”. 


\section{Instituciones objeto de comparación}

Los centros analizados en este estudio (CNRS, HG, MPG, CSIC y CNR) son las cinco instituciones científicas gubernamentales de Europa occidental que en 2015 encabezaron el SCImago Institutions Rankings o SIR (SCImago Lab, 2015). Este ranking, desarrollado por SCImago Lab, resulta ser el más apropiado para la selección de los centros a comparar, pues para su elaboración recurre a Scopus, la misma base de datos empleada para el análisis bibliométrico realizado en este trabajo.

La información obtenida de los informes de actividad que anualmente publican los centros analizados (CNRS, 2017a; HG, 2016; MPG, 2017a; CSIC, 2017a; CNR, 2016), así como de sus respectivos sitios web oficiales (CNRS, 2017b; HG, 2017; MPG, 2017b; CSIC, 2017b; CNR, 2017) permite presentarlos preliminarmente al análisis bibliométrico.

El CSIC es una institución pública con forma jurídica de Agencia Estatal ligada al Ministerio de Economía y Competitividad. Fundado en 1939, está formado por una red de 122 institutos repartidos por toda la geografía española, excepto la Escuela Española de Historia y Arqueología, que se encuentra en Roma (Italia). En el CSIC trabajan aproximadamente 15.000 personas, siendo 3.000 de ellas personal de investigación con contrato permanente, y otras tantas personal de investigación en formación. El presupuesto en 2016 ascendió a 724,3 millones de euros.

El Centre National de la Recherche Scientifique (CNRS) es un organismo público de investigación coordinado por el Ministerio de Enseñanza Superior, Investigación e Innovación. También fundado oficialmente en 1939, reúne en la actualidad a más de 32.000 personas. El personal de investigación asciende a más de 11.000. Su actividad se lleva a cabo en más de 1.100 centros de investigación que son gestionados por 10 grandes institutos. En 2016 contó con un presupuesto de 3.200 millones de euros.

El Helmholtz-Gemeinschaft (HG) fue creado en 1995. A diferencia de los otros cuatro centros de investigación estudiados en este trabajo, el centro Helmholtz sólo desarrolla su actividad en seis campos del conocimiento: energía; tierra y medio ambiente; salud; aeronáutica, espacio y transporte; materia; y tecnología clave. Está compuesto por 18 centros de investigación, en los que trabajan más de 38.000 personas. Su presupuesto anual ronda los 4.000 millones de euros.

El Max-Planck-Gesellschaft, fundado en 1948, realiza investigaciones en todas las ramas de la ciencia (ciencias naturales, ciencias de la vida, ciencias sociales y humanidades), centrándose especialmente en los campos más desconocidos. Su infraestructura cuenta actualmente con 83 institutos, 5 de ellos asentados fuera de Alemania (4 en Europa y 1 en Estados Unidos), cada uno de los cuales pertenece a uno de los siguientes tres campos de investigación: Química, física y tecnología; Biología y medicina; y Humanidades. En él trabajan alrededor de 22.000 personas, de entre las cuales, más de 13.000 son personal de investigación. En 2016, su presupuesto ascendió a los 1.800 millones de euros.

El Consiglio Nazionale delle Ricerche (CNR), fundado en 1923, es un organismo público de investigación dependiente del Ministerio de Educación, Universidad e Investigación. En el CNR se llevan a cabo investigaciones en todos 
los campos del conocimiento. Los 7 departamentos que lo componen gestionan un total de 102 institutos en los que trabajan unas 8.000 personas. El organismo italiano cuenta también con más de 300 sedes secundarias localizadas en diferentes puntos del mundo. Su presupuesto anual suele ser de unos 900 millones de euros.

Tabla 1. Datos generales comparados de los centros CNRS, Helmholtz, Max Planck, CSIC y CNR

\begin{tabular}{|c|c|c|c|c|c|}
\hline Datos generales & CNRS & HG & MPG & CSIC & CNR \\
\hline Fundación & 1939 & 1995 (1887) & $\begin{array}{l}1948 \\
(1911)\end{array}$ & $\begin{array}{l}1939 \\
(1907)\end{array}$ & 1923 \\
\hline \multirow{3}{*}{$\begin{array}{l}\text { Áreas de investigación } \\
\text { abarcadas }\end{array}$} & \multirow{3}{*}{ Todas } & Ciencias naturales & \multirow{3}{*}{ Todas } & \multirow{3}{*}{ Todas } & \multirow{3}{*}{ Todas } \\
\hline & & Ciencias de la salud & & & \\
\hline & & Tecnología & & & \\
\hline Presupuesto anual 2016 & $\begin{array}{l}3.200 \\
\mathrm{M} €\end{array}$ & 4.000 M€ & $1.800 \mathrm{M} €$ & $724 \mathrm{M} €$ & $\begin{array}{l}900 \\
\mathrm{M} €\end{array}$ \\
\hline Número de trabajadores & 32.000 & 38.000 & 22.000 & 15.000 & 8.000 \\
\hline $\begin{array}{l}\text { Posición mundial según el } \\
\text { SCImago Institutions } \\
\text { Rankings }\end{array}$ & 1 & 6 & 10 & 13 & 41 \\
\hline $\begin{array}{l}\text { Posición europea según el } \\
\text { SCImago Institutions } \\
\text { Rankings }\end{array}$ & 1 & 2 & 3 & 4 & 5 \\
\hline
\end{tabular}

Como se aprecia en la Tabla 1, el centro Helmholtz es el organismo científico que cuenta con la mayor plantilla (38.000 empleados) y el presupuesto más elevado (4.000 millones de euros). El presupuesto más reducido es el del CSIC (724 millones de euros; más de cinco veces inferior a la del centro Helmholtz), y la plantilla más pequeña es la del CNR (8.000 empleados; casi la mitad menos que su competidor más cercano, el CSIC, y cuatro veces menos que la del CNRS).

\section{Metodología}

Los datos empleados para la elaboración de este estudio fueron obtenidos de Scopus en marzo de 2017.

\subsection{Estrategia de búsqueda}

Para la obtención desde Scopus de los datos sobre producción científica de los cinco centros de investigación analizados en este trabajo se procedió del siguiente modo:

- Se accedió al campo de búsqueda por afiliación o affiliation search (Scopus $>$ Search > Affiliation).

- En la casilla affiliation name se introdujo el nombre o las siglas de la institución científica que se pretendía localizar. 
- Para corroborar que las afiliaciones devueltas por Scopus pertenecían al centro de investigación que se quiere estudiar, se fueron inspeccionando una a una, y se seleccionaron las adecuadas.

- Se accedió a todos los registros (documentos indexados) correspondientes a estas afiliaciones mediante la opción show all documents.

- Todos estos registros fueron filtrados desde la opción analyze search results para el intervalo de tiempo comprendido entre 2006 y 2015.

- Mediante la opción export the data to a CSV file, se descargaron los datos correspondientes a: año (year), tipología documental (document type), idiomas (language), país/territorio (country/territory) y áreas de investigación (subject area).

Para descargar los datos relativos a citas (impacto) se repitieron los primeros cuatro pasos descritos en el apartado anterior y a continuación:

- Se seleccionaron los registros correspondientes a 2006 y se exportaron a un archivo CSV mediante la opción view citation overview.

- Se repitió el mismo proceso para cada uno de los años de la década 2006$2015^{3}$.

Siguiendo este procedimiento se han podido recuperar de Scopus los datos reflejados en la Tabla 2:

Tabla 2. Número de afiliaciones y de registros recuperados en Scopus correspondientes a los centros CNRS, HG, MPG, CSIC y CNR

\begin{tabular}{|l|l|l|l|l|l|}
\hline & CNRS & HG & MPG & CSIC & CNR \\
\hline $\mathrm{N}^{\circ}$ de afiliaciones recuperadas & 501 & 13 & 116 & 160 & 94 \\
\hline $\mathrm{N}^{\text {o de afiliaciones seleccionadas }}$ & 480 & 12 & 115 & 159 & 93 \\
\hline $\mathrm{N}^{0}$ total de registros & 422.247 & 95.027 & 275.378 & 160.506 & 153.237 \\
\hline $\begin{array}{l}\mathrm{N}^{0} \text { de registros de la década 2006- } \\
2015\end{array}$ & 207.049 & 46.472 & 115.236 & 87.359 & 70.845 \\
\hline
\end{tabular}

Cabe añadir, para completar este apartado metodológico, la existencia de problemas y posibles errores a la hora de aislar la producción institucional en los índices de citas. Van Raan (2005) apunta a la multiplicidad de denominaciones con las que se hace constar la filiación en los índices de citas, concretamente WoS, como una limitación importante de estos para análisis bibliométricos institucionales. Más recientemente, buscando las universidades chilenas en Scopus, Krauskopf (2017) obtenía resultados de producción institucional diferentes dependiendo de la estrategia de búsqueda adoptada (por país o por filiación). Con

3 Estos datos se han tenido que descargar en varias fases, año por año, debido a que Scopus sólo permite descargar datos sobre citación de un máximo de 20.000 registros por vez. En el caso del CNRS, se han debido realizar dos descargas para conseguir los datos de 2011, 2012, 2013, 2014 y 2015, pues el número de registros de estos años era superior a 20.000 . 
respecto a los grandes centros de investigación como el CNRS o la Academia China de las Ciencias recopilados en el SCImago Ranking, Mesnard (2012) destaca problemas como las múltiples nomenclaturas o la doble afiliación, que se produce cuando ciertas publicaciones se adscriben a dos o más instituciones. La posibilidad de estos errores representa una importante limitación del trabajo, aunque sea compartida por los cinco centros analizados.

\subsection{Indicadores bibliométricos}

Existen numerosos tipos de indicadores bibliométricos, así como diferentes clasificaciones de los mismos (Spinak, 1998; van Raan, 2004). Los empleados en este trabajo (Tabla 3) son los que suele utilizar el propio CSIC en los informes que realiza anualmente (Bordons, y otros, 2016; González-Albo y otros, 2012).

Tabla 3. Indicadores bibliométricos empleados

Tipo

Actividad

Impacto

Colaboración

\section{Denominación}

Número de publicaciones

Índice de actividad (IA)

Número de citas por publicación

Porcentaje de publicaciones no citadas

Índice $h$

Índice de inmediatez

Frecuencia de colaboración internacional

Índice de colaboración internacional (ICI)

\section{a) Indicadores de actividad}

Los indicadores de actividad miden la producción científica de modo estrictamente cuantitativo. Los empleados en este trabajo incluyen:

- Número de publicaciones. Los datos obtenidos se han clasificado por áreas de investigación, además de observar su evolución en el tiempo.

- Índice de actividad (IA). Sirve para comparar la producción de una institución en una disciplina o área de investigación concreta con la del resto de centros estudiados. Se calcula del siguiente modo:

$$
I A=\frac{\% \text { de publicaciones que una institución dedica a un área }}{\% \text { de publicaciones que todas las instituciones dedican a dicha área }}
$$

Se considera que una institución está especializada en un área determinada cuando al calcular el IA para dicha materia se obtiene como resultado un valor igual o superior a la unidad (1) (González-Albo y otros, 2012). 


\section{b) Indicadores de impacto}

Los indicadores de impacto pretenden medir la visibilidad e influencia de los trabajos de investigación. Por esta condición, y aunque se basen en datos cuantificables (citas, en este caso), se considera que aportan un matiz relativamente cualitativo (Rivera-Hernández, 2015). En este trabajo se han utilizado los siguientes:

- Número de citas por publicación. El número total de citas recibidas por una entidad es en sí un indicador. A partir del mismo es posible obtener datos adicionales como porcentajes y promedio de citas por documento.

- Porcentaje de publicaciones no citadas. Resulta interesante conocer los datos que se pueden obtener con este indicador teniendo en cuenta que la probabilidad de que un estudio nunca sea citado es muy alta (Hamilton, 1990; Seglen, 1997), incluso habiendo sido sometido a una revisión por pares (Aksnes y Taxt, 2004). Que un trabajo no haya sido citado no significa que sea de baja calidad (Velasco y otros, 2012). Además, MacRoberts (2010) ha demostrado que la mayoría de los investigadores no citan en sus trabajos todos los estudios que han influido en sus investigaciones.

- Índice h. "Un autor tiene índice h si h de sus $\mathrm{N}$ publicaciones recibe al menos h citas cada uno, y los otros (N-h) trabajos tienen como máximo h citas cada uno” (Hirsch, 2005). Aunque en la definición original, Hirsch no propone explícitamente calcular el Índice h para una institución sino para una persona, este indicador puede emplearse para medir el impacto de un autores, revistas, centros de investigación (como en este caso) e incluso países. Este enunciado se puede representar mediante la siguiente función (Torra y Narukawa, 2008; Beliakov y otros, 2016: 176):

$$
\text { Índice } h f(x)=\max _{x} \min (f(x), x) \quad f(x)=\text { número de citas de cada publicación }
$$

- Índice de inmediatez. Este indicador permite conocer la frecuencia con la que se cita un trabajo de investigación en el mismo año en que ha sido publicado. Es decir, mide la velocidad con la que una publicación es citada desde su aparición. Se puede calcular utilizando la siguiente fórmula (Andrés, 2009: 82):

Número de citas que determinados trabajos Índice de inmediatez $=\frac{\text { recibieron el mismo año en el que fueron publicados }}{\text { Número de trabajos publicados en dicho año }}$

Hay que tener en cuenta que en este estudio las citas se han calculado con una ventana de citación variable, que abarca el periodo comprendido entre la fecha de publicación de los trabajos más antiguos (enero de 2006) y la fecha de descarga de los datos (marzo de 2017). Como es lógico, esto supone que los trabajos más antiguos han tenido mayores posibilidades de ser citados. Esto influirá a la hora de calcular los indicadores anteriormente descritos, pero no será un inconveniente para comparar los centros de investigación aquí analizados, ya que este factor afectará de igual manera a todos ellos. 


\section{c) Indicadores de colaboración}

Los utilizados en este trabajo comprenden:

- Frecuencia de colaboración internacional. Número de veces que un país, o cualquier institución de investigación de dicho país, aparece como coautor en los estudios publicados por un determinado centro de investigación. Las cifras obtenidas se pueden presentar como tal o en porcentajes. Este indicador podría ser considerado como un subtipo de indicador de actividad, pues está basado únicamente en datos brutos de producción.

- Índice de colaboración internacional (ICI). Permite conocer cómo de fuerte es la relación de colaboración que un centro de investigación mantiene con un determinado país en comparación con la que el resto de instituciones estudiadas mantienen con el mismo país. Está inspirado en el índice de colaboración propuesto por Andrés (2009: 41), aunque se calcula de manera similar al índice de actividad. Su fórmula es la siguiente:

$$
\text { ICI }=\frac{\begin{array}{c}
\% \text { de ocasiones en las que un país ha colaborado } \\
\text { con determinado centro de investigación }
\end{array}}{\text { \% de ocasiones en las que dicho país ha colaborado }}
$$

Se considera que la relación de colaboración mantenida entre determinado centro de investigación y cierto país es especialmente significativa cuando al calcular el indicador ICI se obtiene un valor igual o superior a 1.

\section{Resultados}

\subsection{Actividad}

\subsubsection{Evolución de la producción}

De los trabajos publicados por el CSIC durante la década comprendida entre 2006 y 2015, 87.359 han sido incluidos en la base de datos de Scopus (Tabla 4). A lo largo de este periodo de tiempo, la producción ha ido creciendo con relativa constancia, situándose el incremento medio anual en 0,34\%. Solamente en los años 2013 y 2015 , se ha producido un crecimiento negativo $(-0,10 \%$ y $-1,03 \%$ respectivamente). El año 2014, en el que se publicaron 10.133 documentos, ha sido el más productivo de la década. 
Tabla 4. Evolución anual de la producción científica de los centros

CNRS, HG, MPG, CSIC y CNR

\begin{tabular}{|c|c|c|c|c|c|c|c|c|c|c|}
\hline \multirow[b]{2}{*}{ 旁 } & \multicolumn{2}{|c|}{ CNRS } & \multicolumn{2}{|c|}{ HG } & \multicolumn{2}{|c|}{ MPG } & \multicolumn{2}{|c|}{ CSIC } & \multicolumn{2}{|c|}{ CNR } \\
\hline & $\stackrel{\circ}{\circ}$ & 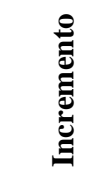 & $\stackrel{\circ}{\circ}$ & 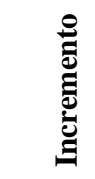 & $\stackrel{\mathscr{O}}{\circ}$ & 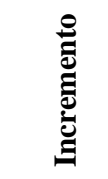 & ڤ̆ & 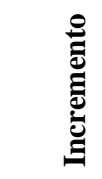 & $\stackrel{\mathscr{O}}{\circ}$ & 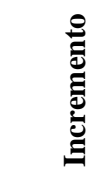 \\
\hline 2006 & 15.471 & $(-)$ & 3.544 & $(-)$ & 9.748 & $(-)$ & 6.574 & $(-)$ & 5.643 & $(-)$ \\
\hline 2007 & 16.664 & $0,58 \%$ & 3.797 & $0,54 \%$ & 10.086 & $0,29 \%$ & 7.236 & $0,76 \%$ & 5.973 & $0,47 \%$ \\
\hline 2008 & 17.189 & $0,25 \%$ & 4.020 & $0,48 \%$ & 10.463 & $0,33 \%$ & 7.629 & $0,45 \%$ & 6.047 & $0,10 \%$ \\
\hline 2009 & 18.547 & $0,66 \%$ & 4.218 & $0,43 \%$ & 10.944 & $0,42 \%$ & 8.098 & $0,54 \%$ & 6.240 & $0,27 \%$ \\
\hline 2010 & 19.607 & $0,51 \%$ & 4.521 & $0,65 \%$ & 11.457 & $0,45 \%$ & 9.006 & $1,04 \%$ & 6.334 & $0,13 \%$ \\
\hline 2011 & 20.926 & $0,64 \%$ & 4.717 & $0,42 \%$ & 12.233 & $0,67 \%$ & 9.390 & $0,44 \%$ & 6.939 & $0,85 \%$ \\
\hline 2012 & 23.045 & $1,02 \%$ & 4.997 & $0,60 \%$ & 12.743 & $0,44 \%$ & 10.071 & $0,78 \%$ & 7.671 & $1,03 \%$ \\
\hline 2013 & 24.839 & 0,87\% & 5.376 & 0,82\% & 12.617 & $-0,11 \%$ & 9.988 & $-0,10 \%$ & 8.432 & $1,07 \%$ \\
\hline 2014 & 25.645 & 0,39\% & 5.593 & $0,47 \%$ & 12.584 & $-0,03 \%$ & 10.133 & $0,17 \%$ & 8.565 & $0,19 \%$ \\
\hline 2015 & 25.116 & $-0,26 \%$ & 5.689 & $0,21 \%$ & 12.361 & $-0,19 \%$ & 9.234 & $-1,03 \%$ & 9.001 & $0,62 \%$ \\
\hline Total & 207.049 & $(-)$ & 46.472 & $(-)$ & 115.236 & $(-)$ & 87.359 & $(-)$ & 70.845 & $(-)$ \\
\hline$\%$ & $39,29 \%$ & $(-)$ & $8,82 \%$ & $(-)$ & $21,87 \%$ & $(-)$ & $16,58 \%$ & $(-)$ & $\begin{array}{l}13,44 \\
\%\end{array}$ & $(-)$ \\
\hline
\end{tabular}

En comparación con los otros centros estudiados (Gráfico 1), el CSIC es el tercer centro con mayor cantidad de documentos indexados por Scopus. Por delante se encuentran el CNRS francés (con un total de 207.049 documentos) y el Max Planck alemán (con 115.236 documentos). El cuarto y el quinto lugar lo ocupan respectivamente el CNR italiano y el Helmholtz alemán, habiendo publicado en revistas recopiladas en Scopus respectivamente 70.845 y 46.472 documentos. 
Gráfico 1. Producción científica de los centros CNRS, HG, MPG, CSIC y CNR

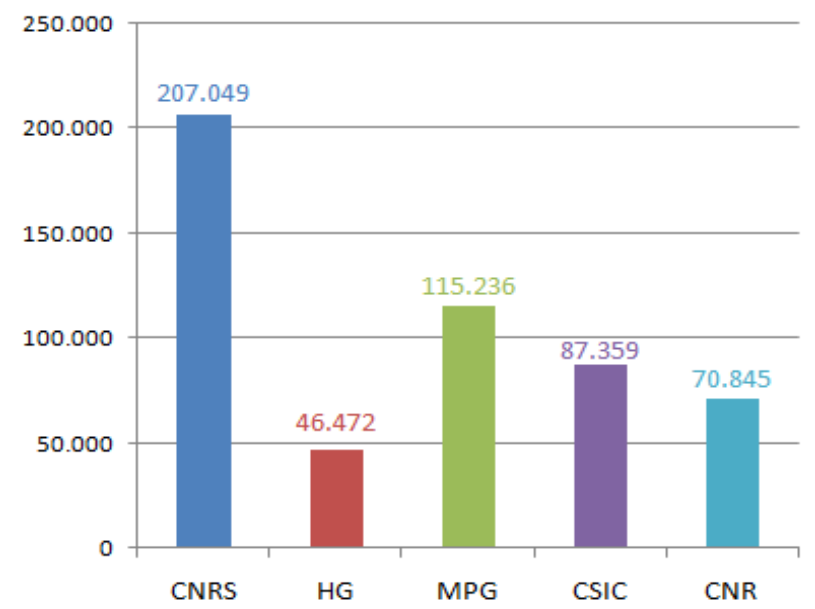

Desde el punto de vista de la evolución de la producción a lo largo de la década (Gráfico 2), el crecimiento anual de la producción del CNRS es visiblemente más acusado que el del resto de centros de investigación, situándose el punto de mayor productividad en el año 2014, al igual que en el caso del CSIC. Los centros Helmholtz y CNR son los únicos cuya producción crece ininterrumpidamente a lo largo de la década analizada. El centro Max Planck es el único que ha encadenado varios años de crecimiento negativo, entre 2012 y 2015.

Gráfico 2. Evolución anual de la producción científica de los centros CNRS, HG, MPG, CSIC y CNR

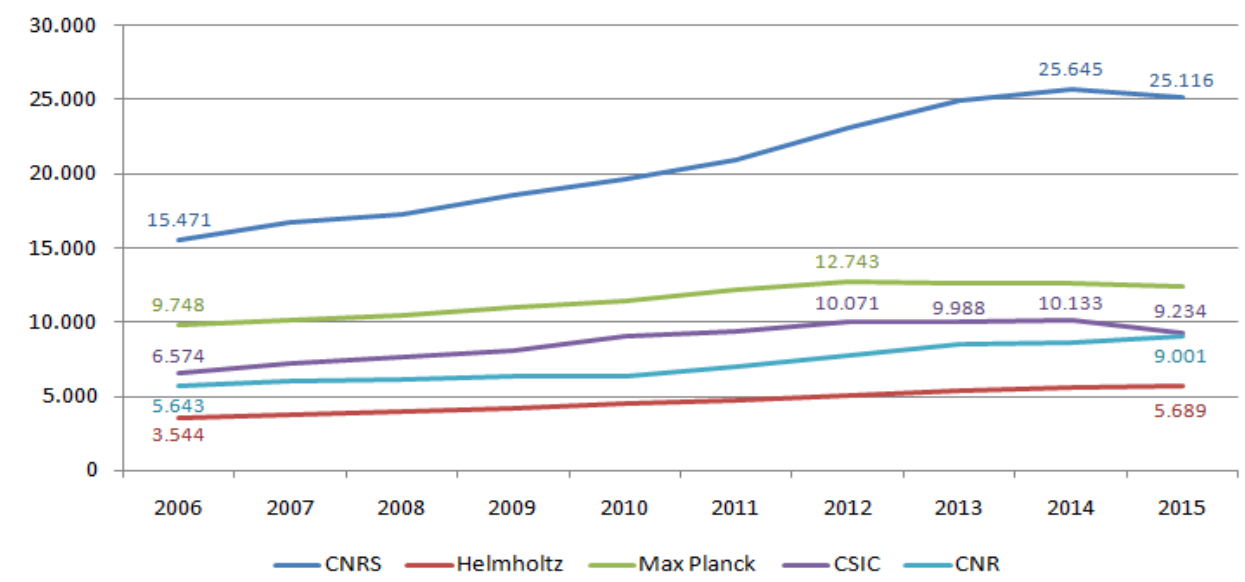

Sería posible profundizar más en el análisis de la producción de estos cinco centros clasificando sus publicaciones según el tipo de documento y el idioma empleados. Pero dado que los resultados a obtener son previsibles (el artículo y el 
inglés son respectivamente el tipo de documento y el idioma empleados por antonomasia), se ha optado por omitirlos en el cuerpo de este trabajo. A este respecto sólo se señalarán los dos datos siguientes:

- Todas las instituciones emplean el inglés en más del 95\% de sus trabajos.

- El formato artículo representa más del $90 \%$ de la producción de los cinco centros.

\subsection{2. Áreas de investigación}

Para la catalogación de sus registros, Scopus distingue entre 27 áreas de investigación (García y otros, 2011) y las organiza en cuatro categorías: Ciencias físicas, Ciencias de la vida, Ciencias de la salud y Ciencias sociales y humanidades (Scopus, 2006). El desglose de la producción por materias que presentamos aquí reproduce la clasificación de Scopus en áreas de investigación y categorías.

La Tabla 5 muestra en cuáles áreas de investigación se reparte la producción global de los cinco centros y la Tabla 4 los respectivos índices de actividad. Para comprender los datos ofrecidos en las Tablas de 5 a 8 es importante recordar que Scopus puede asignar un mismo documento a varias áreas de investigación. Esto explica que las cifras que aparecen en las filas de totales de las Tablas 5 y 7 sean superiores al número total de documentos publicados por cada centro.

Tabla 5. Producción científica de los centros CNRS, HG, MPG, CSIC y CNR según las áreas de investigación* y **

\begin{tabular}{|c|c|c|c|c|c|c|c|c|c|c|}
\hline \multirow[b]{2}{*}{$\begin{array}{l}\text { Áreas de } \\
\text { investigación }\end{array}$} & \multicolumn{2}{|c|}{ CNRS } & \multicolumn{2}{|c|}{ HG } & \multicolumn{2}{|c|}{ MPG } & \multicolumn{2}{|c|}{ CSIC } & \multicolumn{2}{|c|}{ CNR } \\
\hline & 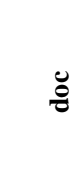 & 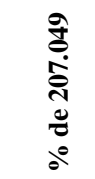 & $\stackrel{\ddot{\theta}}{0}$ & 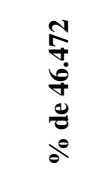 & $\mathscr{~}$ & 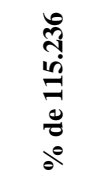 & $\mathscr{\Xi}$ & 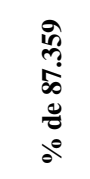 & $\ddot{\theta}$ & 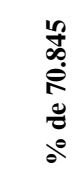 \\
\hline $\begin{array}{l}\text { Física y } \\
\text { astronomía }\end{array}$ & 63.161 & $30,51 \%$ & 12.304 & $26,48 \%$ & 46.903 & $40,70 \%$ & 20.125 & $23,04 \%$ & 16.327 & $23,05 \%$ \\
\hline $\begin{array}{l}\text { Bioquímica, } \\
\text { genética y } \\
\text { biología } \\
\text { molecular }\end{array}$ & 29.825 & $14,40 \%$ & 7.327 & $15,77 \%$ & 22.346 & $19,39 \%$ & 17.226 & $19,72 \%$ & 12.463 & $17,59 \%$ \\
\hline $\begin{array}{l}\text { Ciencia de } \\
\text { materiales }\end{array}$ & 31.059 & $15,00 \%$ & 6.005 & $12,92 \%$ & 15.332 & $13,30 \%$ & 11.749 & $13,45 \%$ & 11.452 & $16,16 \%$ \\
\hline Química & 28.410 & $13,72 \%$ & 4.535 & $9,76 \%$ & 15.152 & $13,15 \%$ & 14.429 & $16,52 \%$ & 11.181 & $15,78 \%$ \\
\hline Ingenieria & 35.927 & $17,35 \%$ & 4.188 & $9,01 \%$ & 8.943 & $7,76 \%$ & 8.988 & $10,29 \%$ & 11.762 & $16,60 \%$ \\
\hline Medicina & 23.552 & $11,38 \%$ & 6.955 & $14,97 \%$ & 14.527 & $12,61 \%$ & 11.340 & $12,98 \%$ & 11.780 & $16,63 \%$ \\
\hline $\begin{array}{l}\text { Ciencias de la } \\
\text { tierra y el } \\
\text { espacio }\end{array}$ & 21.601 & $10,43 \%$ & 6.235 & $13,42 \%$ & 16.759 & $14,54 \%$ & 10.304 & $11,80 \%$ & 7.669 & $10,83 \%$ \\
\hline $\begin{array}{l}\text { Ciencias } \\
\text { agrícolas y } \\
\text { biológicas }\end{array}$ & 16.073 & $7,76 \%$ & 6.029 & $12,97 \%$ & 10.497 & $9,11 \%$ & 22.320 & $25,55 \%$ & 7.424 & $10,48 \%$ \\
\hline
\end{tabular}




\begin{tabular}{|c|c|c|c|c|c|c|c|c|c|c|}
\hline $\begin{array}{l}\text { Ciencias de la } \\
\text { computación }\end{array}$ & 26.942 & $13,01 \%$ & 1.066 & $2,29 \%$ & 6.678 & $5,80 \%$ & 4.169 & $4,77 \%$ & 9.353 & $13,20 \%$ \\
\hline Matemáticas & 22.768 & $11,00 \%$ & 1.891 & $4,07 \%$ & 7.570 & $6,57 \%$ & 3.105 & $3,55 \%$ & 5.280 & $7,45 \%$ \\
\hline $\begin{array}{l}\text { Ciencias } \\
\text { ambientales }\end{array}$ & 8.674 & $4,19 \%$ & 6.641 & $14,29 \%$ & 3.142 & $2,73 \%$ & 9.211 & $10,54 \%$ & 4.848 & $6,84 \%$ \\
\hline $\begin{array}{l}\text { Ingeniería } \\
\text { química }\end{array}$ & 9.806 & $4,74 \%$ & 1.650 & $3,55 \%$ & 5.454 & $4,73 \%$ & 5.431 & $6,22 \%$ & 4.087 & $5,77 \%$ \\
\hline $\begin{array}{l}\text { Inmunología y } \\
\text { microbiología }\end{array}$ & 6.500 & $3,14 \%$ & 2.737 & $5,89 \%$ & 3.718 & $3,23 \%$ & 5.258 & $6,02 \%$ & 1.844 & $2,60 \%$ \\
\hline $\begin{array}{l}\text { Ciencias } \\
\text { sociales }\end{array}$ & 8.527 & $4,12 \%$ & 673 & $1,45 \%$ & 3.840 & $3,33 \%$ & 2.278 & $2,61 \%$ & 1.729 & $2,44 \%$ \\
\hline Neurociencia & 5.297 & $2,56 \%$ & 656 & $1,41 \%$ & 6.341 & $5,50 \%$ & 1.725 & $1,97 \%$ & 2.245 & $3,17 \%$ \\
\hline Energía & 3.992 & $1,93 \%$ & 1.571 & $3,38 \%$ & 2.361 & $2,05 \%$ & 1.914 & $2,19 \%$ & 2.058 & $2,90 \%$ \\
\hline Farmacia & 4.187 & $2,02 \%$ & 1.314 & $2,83 \%$ & 1.161 & $1,01 \%$ & 2.264 & $2,59 \%$ & 2.451 & $3,46 \%$ \\
\hline $\begin{array}{l}\text { Artes y } \\
\text { humanidades }\end{array}$ & 6.001 & $2,90 \%$ & 285 & $0,61 \%$ & 2.420 & $2,10 \%$ & 1.637 & $1,87 \%$ & 861 & $1,22 \%$ \\
\hline Multidisciplinar & 2.818 & $1,36 \%$ & 660 & $1,42 \%$ & 3.157 & $2,74 \%$ & 1.049 & $1,20 \%$ & 608 & $0,86 \%$ \\
\hline Psicología & 2.114 & $1,02 \%$ & 1.104 & $2,38 \%$ & 3.062 & $2,66 \%$ & 163 & $0,19 \%$ & 689 & $0,97 \%$ \\
\hline $\begin{array}{l}\text { Economía, } \\
\text { econometría y } \\
\text { finanzas }\end{array}$ & 1.826 & $0,88 \%$ & 1.035 & $2,23 \%$ & 877 & $0,76 \%$ & 287 & $0,33 \%$ & 192 & $0,27 \%$ \\
\hline $\begin{array}{l}\text { Negocios, } \\
\text { administración } \\
\text { y contabilidad }\end{array}$ & 1.400 & $0,68 \%$ & 1.629 & $3,51 \%$ & 560 & $0,49 \%$ & 246 & $0,28 \%$ & 353 & $0,50 \%$ \\
\hline $\begin{array}{l}\text { Ciencias de la } \\
\text { decisión }\end{array}$ & 1.963 & $0,95 \%$ & 165 & $0,36 \%$ & 420 & $0,36 \%$ & 278 & $0,32 \%$ & 583 & $0,82 \%$ \\
\hline $\begin{array}{l}\text { Profesiones de } \\
\text { la salud }\end{array}$ & 853 & $0,41 \%$ & 930 & $2,00 \%$ & 380 & $0,33 \%$ & 160 & $0,18 \%$ & 400 & $0,56 \%$ \\
\hline Enfermería & 448 & $0,22 \%$ & 823 & $1,77 \%$ & 112 & $0,10 \%$ & 575 & $0,66 \%$ & 374 & $0,53 \%$ \\
\hline Veterinaria & 348 & $0,17 \%$ & 993 & $2,14 \%$ & 94 & $0,08 \%$ & 615 & $0,70 \%$ & 169 & $0,24 \%$ \\
\hline Odontología & 82 & $0,04 \%$ & 1.475 & $3,17 \%$ & 46 & $0,04 \%$ & 59 & $0,07 \%$ & 100 & $0,14 \%$ \\
\hline Indefinido & 4 & $0,00 \%$ & 3 & $0,01 \%$ & 7 & $0,01 \%$ & 3 & $0,00 \%$ & 11 & $0,02 \%$ \\
\hline Total & 364.158 & $(-)$ & 80.879 & $(-)$ & 201.859 & $(-)$ & 156.908 & $(-)$ & 128.293 & $(-)$ \\
\hline
\end{tabular}

Tabla 6. Índice de actividad (IA) de los centros CNRS, HG, MPG, CSIC y CNR según las áreas de investigación

\begin{tabular}{|l|l|l|l|l|l|}
\hline \multirow{2}{*}{ Áreas de investigación } & \multicolumn{5}{|c|}{ IA } \\
\cline { 2 - 7 } & \multicolumn{1}{|c|}{ CNRS } & HG & MPG & CSIC & CNR \\
\hline Física y astronomía & 1,01 & 0,88 & 1,35 & 0,76 & 0,76 \\
\hline $\begin{array}{l}\text { Bioquímica, genética } \\
\text { biología molecular }\end{array}$ & 0,85 & 0,93 & 1,15 & 1,17 & 1,04 \\
\hline Ciencia de materiales & 1,05 & 0,90 & 0,93 & 0,94 & 1,13 \\
\hline
\end{tabular}




\begin{tabular}{|l|l|l|l|l|l|}
\hline Química & 0,98 & 0,70 & 0,94 & 1,18 & 1,13 \\
\hline Ingeniería & 1,31 & 0,68 & 0,59 & 0,78 & 1,25 \\
\hline Medicina de laerra y el & 0,88 & 1,16 & 0,97 & 1,00 & 1,29 \\
\hline $\begin{array}{l}\text { Ciencias de } \\
\text { espacio }\end{array}$ & 0,88 & 1,13 & 1,22 & 0,99 & 0,91 \\
\hline $\begin{array}{l}\text { Ciencias agrícolas y } \\
\text { biológicas }\end{array}$ & 0,66 & 1,10 & 0,77 & 2,16 & 0,89 \\
\hline Ciencias de la computación & 1,42 & 0,25 & 0,63 & 0,52 & 1,44 \\
\hline Matemáticas & 1,43 & 0,53 & 0,85 & 0,46 & 0,97 \\
\hline Ciencias ambientales & 0,68 & 2,32 & 0,44 & 1,71 & 1,11 \\
\hline Ingeniería química & 0,94 & 0,71 & 0,94 & 1,24 & 1,15 \\
\hline Inmunología y microbiología & 0,82 & 1,55 & 0,85 & 1,58 & 0,68 \\
\hline Ciencias sociales & 1,27 & 0,45 & 1,03 & 0,81 & 0,75 \\
\hline Neurociencia & 0,83 & 0,46 & 1,78 & 0,64 & 1,03 \\
\hline Energía & 0,85 & 1,50 & 0,91 & 0,97 & 1,29 \\
\hline Farmacia & 0,94 & 1,31 & 0,47 & 1,20 & 1,60 \\
\hline Artes y humanidades & 1,36 & 0,29 & 0,99 & 0,88 & 0,57 \\
\hline Multidisciplinar & 0,86 & 0,90 & 1,74 & 0,76 & 0,55 \\
\hline Psicología & 0,75 & 1,76 & 1,96 & 0,14 & 0,72 \\
\hline $\begin{array}{l}\text { Economía, econometría y } \\
\text { finanzas }\end{array}$ & 1,10 & 2,78 & 0,95 & 0,41 & 0,34 \\
\hline $\begin{array}{l}\text { Negocios, administración y } \\
\text { contabilidad }\end{array}$ & 0,85 & 4,41 & 0,61 & 0,35 & 0,63 \\
\hline Ciencias de la decisión & 1,47 & 0,55 & 0,56 & 0,49 & 1,27 \\
\hline Profesiones de la salud & 0,80 & 3,87 & 0,64 & 0,35 & 1,09 \\
\hline Enfermería & 0,49 & 4,00 & 0,22 & 1,49 & 1,19 \\
\hline Veterinaria & 0,40 & 5,07 & 0,19 & 1,67 & 0,57 \\
\hline Odontología & 0,12 & 9,49 & 0,12 & 0,20 & 0,42 \\
\hline Indefinido & 0,36 & 1,21 & 1,14 & 0,65 & 2,92 \\
\hline
\end{tabular}

La cuarta parte del total de los estudios publicados por el CSIC (25,55\%) corresponden a la disciplina Ciencias agrícolas y biológicas, siendo el campo de estudio más productivo del centro de investigación español. Cuenta, además, con un índice de actividad considerablemente alto: 2,16 (Tabla 6).

Física y astronomía representa la segunda disciplina más productiva del CSIC (el 23,04\% de las 87.359 publicaciones del CSIC se adscriben a esta especialidad), aunque su índice de actividad no es especialmente sobresaliente: 0,76. Física y astronomía es el área de investigación al que más dedicación prestan los centros europeos aquí estudiados, exceptuando al CSIC. Aunque el CNRS es el que ha publicado más trabajos relacionados con esta materia (63.161), el centro que vincula a esta especialidad un mayor porcentaje de su producción es el Max Planck: el 40,70\% de sus 115.236 publicaciones. En consecuencia, el índice de 
actividad del centro alemán en esta disciplina también es el más elevado de los cinco centros: 1,35. Hay que señalar que, aunque el CSIC sea el centro que menos porcentaje de su producción relaciona con el área de Física y astronomía, no quiere decir que su aportación a este campo no sea significativa. De hecho, la cantidad de documentos registrados en Scopus con la etiqueta de esta especialidad es mayor para el CSIC que para el CNR o el Helmholtz (CSIC: 20.125; CNR: 16.327; Helmholtz: 12.304).

En Bioquímica, genética y biología molecular, el área de investigación más destacado del grupo de Ciencias de la vida, el CSIC es el centro que más porcentaje de producción le dedica (un 19,72\%), seguido muy de cerca por el Max Planck (19,39\%). El IA de la institución española en esta materia es de 1,17, mientras que el de la alemana es de 1,15. El CNR también obtiene un IA positivo de 1,04 para la misma especialidad. No obstante, pese a no destacar ni en porcentaje de producción ni en IA, quien más estudios aporta a esta materia es el CNRS, con 29.825 documentos registrados en Scopus.

El CNRS es también el centro que más trabajos ha publicado en materia de Medicina, la principal disciplina de Ciencias de la Salud: 23.552. Sin embargo, esta cifra sólo supone el 11,38\% del total de la producción del centro francés (207.049 registros), un porcentaje inferior al obtenido por el resto de centros. El mejor dato según este indicador lo obtiene el CNR, pues el 16,63\% de sus publicaciones están vinculadas al área de Medicina. Gracias a este porcentaje, el centro italiano consigue alcanzar un IA de 1,29, el mayor de los obtenidos por sus homólogos. El centro Helmholtz y el CSIC también consiguieron unos IA positivos de 1,14 y 1,00 respectivamente. El centro Max Planck se quedó a tres décimas de alcanzar la unidad $(0,97)$ y el CNRS con un 0,88 volvió a colocarse en el último puesto.

Es en Ciencias sociales, el área de investigación más destacada de la categoría igualmente denominada Ciencias sociales (y humanidades), donde el CNRS supera al resto de centros en todos los aspectos, pues es el que más estudios ha publicado en este área (8.527), además de ser el que dedica el mayor porcentaje del total de su producción a dicha materia: un 4,12\%. Debido a esto, su IA de 1,27, también resulta ser el más alto. De entre los otros cuatro centros de investigación restantes, sólo el Max Planck cuenta también con un IA medianamente superior a $1(1,03$, para ser exactos).

Es sorprendente el dominio que ejerce el centro Helmholtz sobre una buena parte de las áreas de investigación según los datos obtenidos con el indicador IA, apuntando al alto grado de especialización de este centro (Tabla 6). En 14 de las 27 disciplinas con las que trabaja Scopus, el centro alemán obtiene un índice de actividad igual o superior a 1,00, al igual que el CNR. Lo que verdaderamente hace sobresalir al centro Helmholtz por encima de los otros cuatro es que el IA de 7 de estas disciplinas es especialmente elevado, alcanzando cifras comprendidas entre 2,32 y 9,49 (véase la Tabla 6). Estas materias tan destacadas incluyen: Ciencias ambientales; Economía, econometría y finanzas; Negocios, administración y contabilidad; Profesiones de la salud; Enfermería; Veterinaria; y Odontología. De entre las otras cuatro instituciones restantes, sólo el CSIC logró alcanzar un IA 
superior a 2,00 en alguna disciplina. Lo hizo en Ciencias biológicas y agrícolas, donde obtuvo un 2,16, como ya comentamos.

Si analizamos los datos por categorías (Tabla 7), para todos los centros, la categoría Ciencias Físicas resulta ser la más productiva en términos absolutos, seguida de Ciencias de la vida, Ciencias de la salud y Ciencias sociales (y humanidades). A la primera de ellas, las cinco instituciones dedican más de la mitad de su producción. Al resto, ninguno de los centros dedica al menos un cuarto (25\%) de su producción, excepto el CSIC a Ciencias de la vida, pues el 31,10\% de sus publicaciones pertenecen a dicha categoría.

Tabla 7. Producción científica de los centros CNRS, HG, MPG, CSIC y CNR por categorías de áreas de investigación*

\begin{tabular}{|c|c|c|c|c|c|c|c|c|c|c|}
\hline \multirow{2}{*}{ Categorías } & \multicolumn{2}{|c|}{ CNRS } & \multicolumn{2}{|c|}{ HG } & \multicolumn{2}{|c|}{ MPG } & \multicolumn{2}{|c|}{ CSIC } & \multicolumn{2}{|c|}{ CNR } \\
\hline & doc & $\%$ & doc & $\%$ & doc & $\%$ & doc & $\%$ & doc & $\%$ \\
\hline $\begin{array}{l}\text { Ciencias de la } \\
\text { salud }\end{array}$ & 25.283 & $6,94 \%$ & 11.176 & $13,82 \%$ & 15.159 & $7,51 \%$ & 12.749 & $8,13 \%$ & 12.823 & $10,00 \%$ \\
\hline Ciencias físicas & 252.340 & $69,29 \%$ & 46.086 & $56,98 \%$ & 128.294 & $63,56 \%$ & 89.425 & $56,99 \%$ & 84.017 & $65,49 \%$ \\
\hline $\begin{array}{l}\text { Ciencias } \\
\text { sociales } \\
\text { (y } \\
\text { humanidades) }\end{array}$ & 21.831 & $5,99 \%$ & 4.891 & $6,05 \%$ & 11.179 & $5,54 \%$ & 4.889 & $3,12 \%$ & 4.407 & $3,44 \%$ \\
\hline $\begin{array}{l}\text { Ciencias de la } \\
\text { vida }\end{array}$ & 61.882 & $16,99 \%$ & 18.063 & $22,33 \%$ & 44.063 & $21,83 \%$ & 48.793 & $31,10 \%$ & 26.427 & $20,60 \%$ \\
\hline Multidisciplinar & 2.818 & $0,77 \%$ & 660 & $0,82 \%$ & 3.157 & $1,56 \%$ & 1.049 & $0,67 \%$ & 608 & $0,47 \%$ \\
\hline Indefinido & 4 & $0,00 \%$ & 3 & $0,00 \%$ & 7 & $0,00 \%$ & 3 & $0,00 \%$ & 11 & $0,01 \%$ \\
\hline Total & 364.158 & $100,00 \%$ & 80.879 & $100,00 \%$ & 201.859 & $100,00 \%$ & 156.908 & $100,00 \%$ & 128.293 & $100,00 \%$ \\
\hline
\end{tabular}

Ninguno de los cinco centros de investigación posee un índice de actividad especialmente señalado en las cuatro categorías principales (Tabla 8). Los mejores datos son los del HG: en tres de los cuatro grupos de disciplinas más importantes (Ciencias de la salud, Ciencias sociales y Ciencias de la vida) alcanzó un IA superior a 1, siendo el más elevado el logrado para Ciencias de la salud: 1,64.

El resto de instituciones no destacaron en más de dos categorías. El mejor dato, después del obtenido por el HG en Ciencias de la salud, es el logrado por el CSIC en Ciencias de la vida, categoría en la que el centro español alcanzó un IA de 1,48. 
Tabla 8. Índice de actividad (IA) de los centros CNRS, Helmholtz, Max Planck, CSIC y CNR por categorías de áreas de investigación

\begin{tabular}{|l|l|l|l|l|l|}
\hline \multirow{2}{*}{ Categorías } & \multicolumn{5}{c|}{ IA } \\
\cline { 2 - 7 } & CNRS & HG & MPG & CSIC & CNR \\
\hline Ciencias de la salud & 0,83 & 1,64 & 0,90 & 1,00 & 1,24 \\
\hline Ciencias físicas & 1,07 & 0,87 & 0,98 & 0,90 & 1,04 \\
\hline $\begin{array}{l}\text { Ciencias sociales } \\
\text { humanidades) }\end{array}$ & $\mathbf{1 , 1 8}$ & $\mathbf{1 , 1 8}$ & $\mathbf{1 , 0 8}$ & 0,62 & 0,69 \\
\hline Ciencias de la vida & 0,79 & 1,03 & 1,01 & 1,48 & 0,99 \\
\hline Multidisciplinar & 0,86 & 0,90 & 1,74 & 0,76 & 0,55 \\
\hline Indefinido & 0,36 & 1,21 & 1,14 & 0,65 & 2,92 \\
\hline
\end{tabular}

\subsection{Colaboración}

En la Tabla 9 se muestran los diez países con los que el CSIC colabora más asiduamente. De entre ellos, sólo dos no son europeos: Estados Unidos y México. Alemania, Reino unido, Francia y Estados Unidos encabezan la lista de países más colaboradores con porcentajes de trabajos en colaboración que oscilan entre el 10,05\% de Alemania y el 14,26\% de Estados Unidos. México, país que comparte idioma con España, figuró como coautor en 2.804 publicaciones (el 3,21\% del total de lo producido por el CSIC). Portugal, país vecino, hizo lo mismo en 3.538 investigaciones (el 4,05\%).

Tabla 9. Frecuencia e índice de colaboración internacional (ICI) del CSIC (Top 10)*

\begin{tabular}{|l|l|l|l|}
\hline \multicolumn{1}{|c|}{ Países } & \multicolumn{1}{c|}{ doc } & \multicolumn{1}{c|}{ IC de 87.359 } & \multicolumn{1}{c|}{ ICI } \\
\hline Estados Unidos & 12.457 & $14,26 \%$ & 0,87 \\
\hline Francia & 9.632 & $11,03 \%$ & $\mathbf{1 , 7 6}$ \\
\hline Reino Unido & 9.313 & $10,66 \%$ & $\mathbf{1 , 0 2}$ \\
\hline Alemania & 8.778 & $10,05 \%$ & $\mathbf{1 , 5 5}$ \\
\hline Italia & 7.271 & $8,32 \%$ & $\mathbf{1 , 2 6}$ \\
\hline Suiza & 3.764 & $4,31 \%$ & 0,83 \\
\hline Portugal & 3.538 & $4,05 \%$ & 2,06 \\
\hline Países Bajos & 3.159 & $3,62 \%$ & 0,77 \\
\hline México & 2.804 & $3,21 \%$ & 2,44 \\
\hline Bélgica & 2.541 & $2,91 \%$ & $\mathbf{1 , 0 1}$ \\
\hline Otros & 53.604 & $61,36 \%$ & $(-)$ \\
\hline Total & $\mathbf{1 1 6 . 8 6 1}$ & $\mathbf{( - )}$ & $\mathbf{( - )}$ \\
\hline \multicolumn{2}{|l|}{ *8tudios publicados por el CSIC } \\
\hline
\end{tabular}


Como se ve, Estados Unidos es el país con el que el CSIC establece el mayor número de colaboraciones, pero no por ello su índice de colaboración internacional (ICI) es el más alto. De hecho, es el tercero más bajo. Esto indica que, aunque las relaciones entre el centro de investigación español y EEUU son fuertes, no lo son tanto como las que mantienen las otras instituciones europeas estudiadas en este trabajo (CNRS, HG, MPG y CNR) con el país norteamericano (Tabla 10). Por el contrario, con Portugal y México, el CSIC sí mantiene un vínculo más grande que el mantenido por los mismos países con las otras cuatro instituciones europeas mencionadas. Este dato era previsible teniendo en cuenta que ambos países se encuentran unidos culturalmente con España. El ICI obtenido para el país ibérico es 2,06, y el de México 2,44 (el más elevado de todos).

El Gráfico 3 muestra cómo ha evolucionado la colaboración entre el CSIC y los cinco países más colaboradores. El número de ocasiones en que estos países cooperaron con el centro español se incrementó de manera abrumadora entre 2006 y 2015: casi todos los países mostrados en el gráfico acabaron la década participando en aproximadamente el doble de investigaciones que al principio de la misma.

Gráfico 3. Evolución anual de la frecuencia de colaboración internacional del CSIC (Top 5)

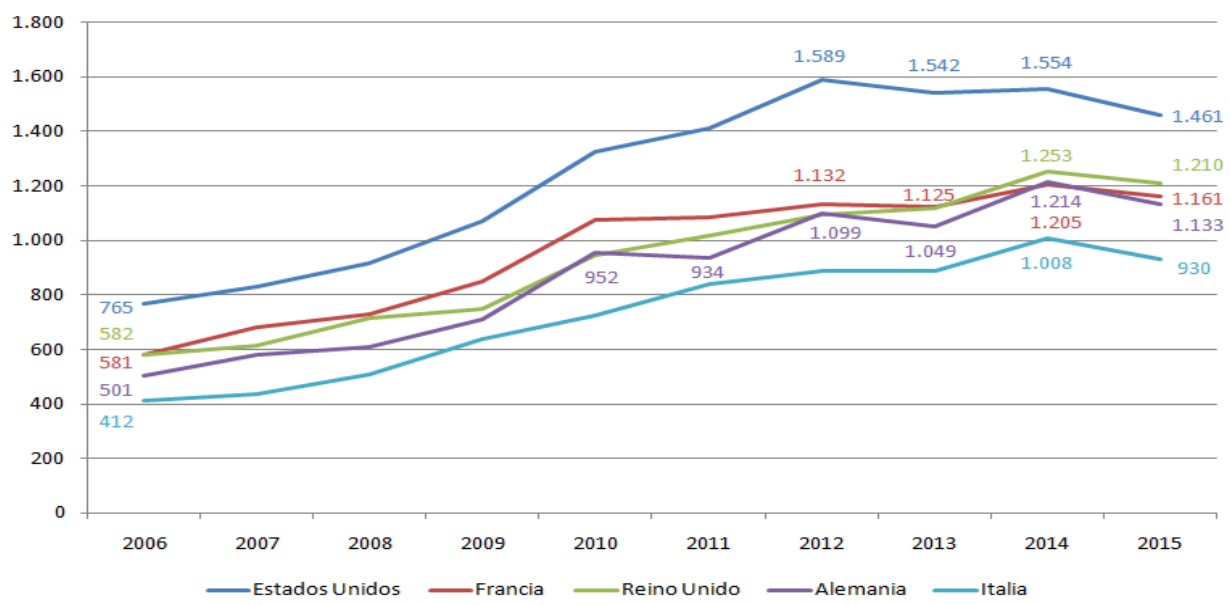

La Tabla 10 muestra los datos de colaboración del CSIC al lado de los otros 4 centros. El país con el que más colaboraron los cinco centros (CNRS, HG, MPG, CSIC y CNR) fue Estado Unidos. El caso más notable fue el del MPG: el país norteamericano participó en más de un cuarto $(25,48 \%)$ de todas las investigaciones publicadas entre 2006 y 2015 por la institución alemana.

Los datos de colaboración más bajos son los del CNR. Sólo cinco países han participado en al menos el 5\% su producción: España, Francia, Alemania, Reino Unido, y EEUU. Ninguno de ellos llegó a alcanzar el 10\%. 
Tabla 10. Frecuencia de colaboración internacional de los centros CNRS, HG, MPG, CSIC y CNR (Top 10)*

\begin{tabular}{|c|c|c|c|c|c|c|c|c|c|c|}
\hline \multirow[b]{2}{*}{ Países } & \multicolumn{2}{|l|}{ CNRS } & \multicolumn{2}{|l|}{ HG } & \multicolumn{2}{|l|}{ MPG } & \multicolumn{2}{|l|}{ CSIC } & \multicolumn{2}{|l|}{ CNR } \\
\hline & ซே & 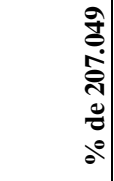 & 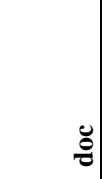 & 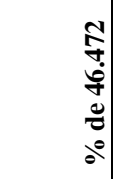 & $\stackrel{\leftrightarrow}{\sigma}$ & 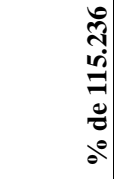 & $\stackrel{\mathscr{o}}{\square}$ & 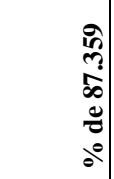 & $\stackrel{\bullet}{*}$ & 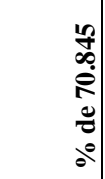 \\
\hline $\begin{array}{l}\text { Estados } \\
\text { Unidos }\end{array}$ & 29.750 & $14,37 \%$ & 7.835 & $16,86 \%$ & 29.362 & $25,48 \%$ & 12.457 & $14,26 \%$ & 6.909 & $9,75 \%$ \\
\hline $\begin{array}{l}\text { Reino } \\
\text { Unido }\end{array}$ & 18.356 & $8,87 \%$ & 5.335 & $11,48 \%$ & 17.061 & $14,81 \%$ & 9.313 & $10,66 \%$ & 4.801 & $6,78 \%$ \\
\hline Italia & 14.874 & $7,18 \%$ & 2.994 & $6,44 \%$ & 9.774 & $8,48 \%$ & 7.271 & $8,32 \%$ & $(-)$ & $(-)$ \\
\hline Alemania & 20.214 & $9,76 \%$ & $(-)$ & $(-)$ & $(-)$ & $(-)$ & 8.778 & $10,05 \%$ & 5.078 & $7,17 \%$ \\
\hline Francia & $(-)$ & $(-)$ & 4.547 & $9,78 \%$ & 13.433 & $11,66 \%$ & 9.632 & $11,03 \%$ & 5.486 & $7,74 \%$ \\
\hline Suiza & 9.444 & $4,56 \%$ & 3.257 & $7,01 \%$ & 8.713 & $7,56 \%$ & 3.764 & $4,31 \%$ & 2.227 & $3,14 \%$ \\
\hline España & 12.567 & $6,07 \%$ & 2.678 & $5,76 \%$ & 8.095 & $7,02 \%$ & $(-)$ & $(-)$ & 3.722 & $5,25 \%$ \\
\hline $\begin{array}{l}\text { Países } \\
\text { Bajos }\end{array}$ & 7.467 & $3,61 \%$ & 2.694 & $5,80 \%$ & 9.503 & $8,25 \%$ & 3.159 & $3,62 \%$ & 2.074 & 2,93\% \\
\hline Rusia & 7.228 & $3,49 \%$ & 2.841 & $6,11 \%$ & 5.880 & $5,10 \%$ & 2.134 & $2,44 \%$ & 1.111 & $1,57 \%$ \\
\hline Canadá & 8.201 & $3,96 \%$ & 1.797 & $3,87 \%$ & 5.149 & $4,47 \%$ & 2.453 & $2,81 \%$ & 1.247 & $1,76 \%$ \\
\hline Otros & 125.756 & $61 \%$ & 32.419 & $70 \%$ & 85.021 & $74 \%$ & 57.900 & $66 \%$ & 22.055 & $31 \%$ \\
\hline Total & 253.857 & $(-)$ & 66.397 & $(-)$ & 191.991 & $(-)$ & 116.861 & $(-)$ & 54.710 & $(-)$ \\
\hline
\end{tabular}

Según los datos de la Tabla 11, se confirma que la institución científica con las relaciones de colaboración internacionales más fuertes es el MPG. Este centro ha obtenido un ICI superior a 1 para los diez países que figuran en la tabla. El vínculo más estrecho lo establece con Francia $(1,87)$.

También son notables las relaciones establecidas por el HG. Sólo con Italia obtiene un ICI inferior a $1(0,97)$. Curiosamente, el ICI más elevado $(1,68)$ lo obtiene por su colaboración con Rusia, un país no perteneciente a la Unión Europea. 
Tabla 11. Índice de colaboración internacional (ICI) de los centros CNRS, HG, MPG, CSIC y CNR (Top 10)

\begin{tabular}{|l|l|l|l|l|l|}
\hline Países & CNRS & HG & MPG & CSIC & CNR \\
\hline Estados Unidos & 0,88 & 1,03 & 1,56 & 0,87 & 0,60 \\
\hline Reino Unido & 0,85 & 1,10 & 1,42 & 1,02 & 0,65 \\
\hline Italia & 1,08 & 0,97 & 1,28 & 1,26 & $(-)$ \\
\hline Alemania & 1,51 & $(-)$ & $(-)$ & 1,55 & 1,11 \\
\hline Francia & $(-)$ & 1,56 & 1,86 & 1,76 & 1,23 \\
\hline Suiza & 0,88 & 1,35 & 1,45 & 0,83 & 0,60 \\
\hline España & 1,18 & 1,12 & 1,37 & $(-)$ & 1,02 \\
\hline Países Bajos & 0,76 & 1,23 & 1,75 & 0,77 & 0,62 \\
\hline Rusia & 0,96 & 1,68 & 1,40 & 0,67 & 0,43 \\
\hline Canadá & 1,11 & 1,08 & 1,25 & 0,79 & 0,49 \\
\hline
\end{tabular}

Los valores alcanzados mediante el indicador ICI son similares para el CNRS y el CSIC. Ambos mantienen relaciones de colaboración destacadas con 4 centros. De sus colaboraciones con Alemania, el centro francés logra su mejor ICI: 1,51. El CSIC también obtiene con el país germano un ICI elevado: 1,55. No obstante, el ICI más alto del centro español $(1,71)$ lo logra por sus relaciones con Francia.

El CNR mantiene sus mejores vínculos con España, Francia y Alemania. Sin embargo, de su colaboración con el resto de países no obtiene nunca un ICI superior a 0,70 , resultando la institución menos colaboradora.

\subsection{Impacto}

En la Tabla 12 se muestra el número de citas que año a año ha ido recibiendo la producción de las cinco instituciones en el periodo 2006-2015.

Tabla 12. Evolución anual de la citación de la producción de los centros CNRS, HG, MPG, CSIC y CNR

\begin{tabular}{|c|c|c|c|c|c|c|c|c|c|c|c|c|c|c|}
\hline & & 2006 & 2007 & 2008 & 2009 & 2010 & 2011 & 2012 & 2013 & 2014 & 2015 & 2016 & 2017 & Total \\
\hline \multirow[b]{2}{*}{ 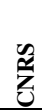 } & Cit & 8.856 & 45.119 & 94.601 & 155.403 & 220.016 & 286.914 & 362.694 & 441.217 & 509.407 & 557.131 & 579.032 & 130.552 & 3.390 .942 \\
\hline & Incr & $(-)$ & $1,07 \%$ & $1,46 \%$ & $1,79 \%$ & $1,91 \%$ & $1,97 \%$ & $2,23 \%$ & $2,32 \%$ & $2,01 \%$ & $1,41 \%$ & $0,65 \%$ & $\begin{array}{l}- \\
13,23 \% \\
\end{array}$ & $(-)$ \\
\hline \multirow[b]{2}{*}{ U } & Cit & 2.288 & 11.158 & 24.280 & 40.788 & 59.633 & 79.184 & 100.562 & 123.759 & 145.721 & 160.126 & 173.411 & 40.688 & 961.598 \\
\hline & Incr & $(-)$ & $0,92 \%$ & $1,36 \%$ & $1,72 \%$ & $1,96 \%$ & $2,03 \%$ & $2,22 \%$ & $2,41 \%$ & $2,28 \%$ & $1,50 \%$ & $1,38 \%$ & $\begin{array}{l}- \\
13,80 \% \\
\end{array}$ & $(-)$ \\
\hline \multirow[b]{2}{*}{ v } & Cit & 9.840 & 46.614 & 93.748 & 152.346 & 210.878 & 278.824 & 354.192 & 413.735 & 473.521 & 503.620 & 515.712 & 115.837 & 3.168.867 \\
\hline & Incr & $(-)$ & $1,16 \%$ & $1,49 \%$ & $1,85 \%$ & $1,85 \%$ & $2,14 \%$ & $2,38 \%$ & $1,88 \%$ & $1,89 \%$ & $0,95 \%$ & $0,38 \%$ & $\begin{array}{l}- \\
12,62 \% \\
\end{array}$ & $(-)$ \\
\hline ज़ & Cit & 3.739 & 20.505 & 45.086 & 76.146 & 110.004 & 150.573 & 192.219 & 229.532 & 266.476 & 287.429 & 297.597 & 70.054 & 1.749 .360 \\
\hline
\end{tabular}




\begin{tabular}{|c|c|c|c|c|c|c|c|c|c|c|c|c|c|c|}
\hline & Incr & $(-)$ & 0,96\% & $1,41 \%$ & $1,78 \%$ & $1,94 \%$ & $2,32 \%$ & $2,38 \%$ & $2,13 \%$ & $2,11 \%$ & $1,20 \%$ & $0,58 \%$ & $\begin{array}{l}- \\
13,01 \% \\
\end{array}$ & $(-)$ \\
\hline & Cit & 2.552 & 14.274 & 30.081 & 48.964 & 67.343 & 86.683 & 109.689 & 135.326 & 160.895 & 177.713 & 188.269 & 43.771 & 1.065 .560 \\
\hline 艺 & Incr & $(-)$ & $1,10 \%$ & $1,48 \%$ & $1,77 \%$ & $1,72 \%$ & $1,82 \%$ & $2,16 \%$ & $2,41 \%$ & $2,40 \%$ & $1,58 \%$ & 0,99\% & $\begin{array}{l}- \\
13,56 \%\end{array}$ & $(-)$ \\
\hline
\end{tabular}

Entre 2006 y 2016 (2017 no se debe tener en cuenta, pues sólo se conocen los datos de sus primeros tres meses), la citación de los cinco centros crece ininterrumpidamente, sufriendo una desaceleración a partir del octavo o noveno año: el crecimiento de los centros CNRS, HG y CNR comienza a frenar en 2014, mientras que el del MPG y el del CSIC lo hace en 2013. A partir de entonces, siguen creciendo, pero lo hacen cada vez más lentamente.

Tras los 11 años de crecimiento continuo (2006-2016), los cinco centros han incrementado la citación de su producción de manera similar (entre un 15 y un $18 \%$, aproximadamente). Pese a lo que pueda parecer viendo la evolución de las líneas del Gráfico 4, el MPG fue el centro cuya citación creció con menos fuerza (un 15,96\%). El mayor aumento lo experimentó el HG (un 17,80\%). El incremento del CNR, el CNRS y el CSIC fue del 17,43\%, 16,81\% y 16,80\% respectivamente.

Gráfico 4. Evolución anual de la citación de la producción de los centros

CNRS, HG, MPG, CSIC y CNR

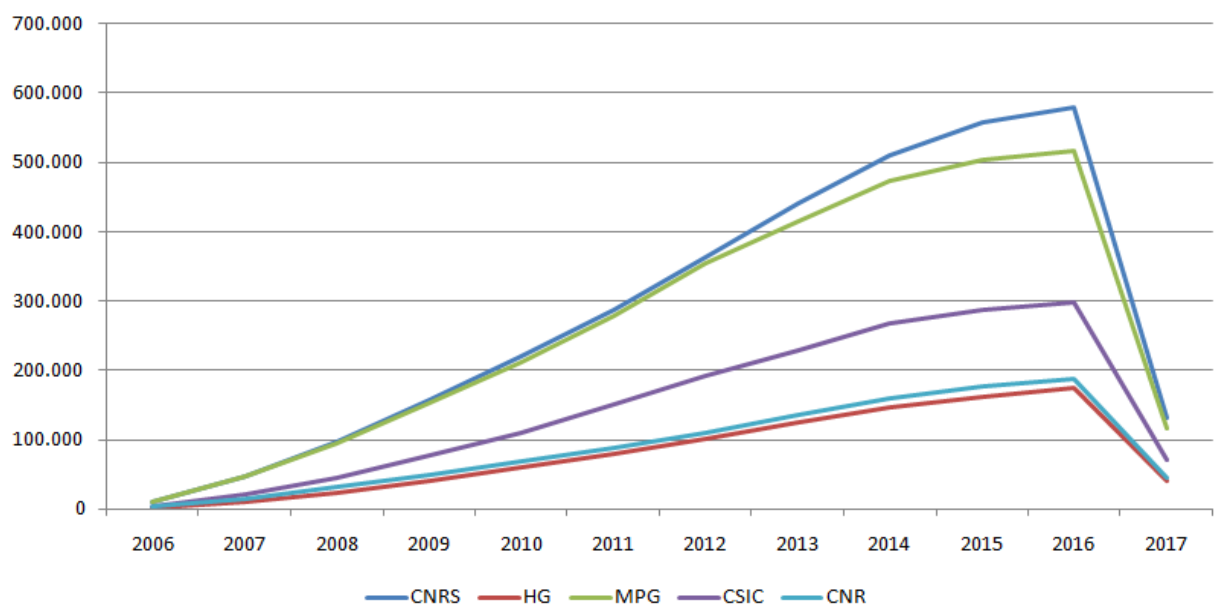

En la última columna de la Tabla 13 se refleja el índice de inmediatez medio que cada centro alcanzó para el total de la producción de la década 2006-2015. El más alto, de 1,22, vuelve a ser el del MPG. Es casi el doble del alcanzado por el CNR $(0,62)$, y aproximadamente un $50 \%$ mayor que el del HG $(0,86)$ y el CISC $(0,80)$. El CNRS sólo logró un 0,72, el segundo valor más bajo. 
Tabla 13. Índice de inmediatez de los centros CNRS, HG, MPG, CSIC y CNR. Evolución anual y promedio

\begin{tabular}{|l|l|l|l|l|l|l|l|l|l|l|l|}
\hline & 2006 & 2007 & 2008 & 2009 & 2010 & 2011 & 2012 & 2013 & 2014 & 2015 & Promedio \\
\hline CNRS & 0,57 & 0,57 & 0,59 & 0,68 & 0,75 & 0,76 & 0,87 & 0,82 & 0,81 & 0,81 & 0,72 \\
\hline HG & 0,65 & 0,62 & 0,70 & 0,83 & 0,92 & 0,83 & 0,93 & 1,10 & 0,97 & 1,07 & 0,86 \\
\hline MPG & 1,01 & 0,99 & 1,00 & 1,16 & 1,29 & 1,25 & 1,48 & 1,43 & 1,36 & 1,28 & 1,22 \\
\hline CSIC & 0,57 & 0,56 & 0,65 & 0,77 & 0,79 & 0,85 & 0,97 & 0,91 & 0,99 & 0,91 & 0,80 \\
\hline CNR & 0,45 & 0,47 & 0,51 & 0,67 & 0,61 & 0,60 & 0,65 & 0,69 & 0,77 & 0,76 & 0,62 \\
\hline
\end{tabular}

En la misma Tabla 13 también se muestra el índice de inmediatez que la producción de cada centro fue obteniendo año a año. Como se observa, las cifras más altas son las del MPG. Su índice de inmediatez fue siempre superior a 1, excepto en 2007, cuando se quedó en 0,99. Los valores alcanzados por los cuatro centros restantes oscilan siempre en valores inferiores al 1, excepto para la producción de 2013 y 2015 del HG, años en que los que el centro alemán obtuvo un índice de inmediatez de 1,10 y 1,07 respectivamente.

Los cincos centros alcanzaron su índice de inmediatez más elevado entre los años 2012 y 2014 (véanse las cifras en cursiva de la Tabla 13). La producción de 2012 del MPG fue la que obtuvo el dato más alto: 1,48. Este valor es casi el doble del alcanzado por el CNR en 2014, año en el que el centro italiano logró su índice de inmediatez más elevado: 0,77. En un punto intermedio entre los valores de estos dos centros se sitúa el mejor dato del CSIC, conseguido también en 2014: 0,99.

Los cinco institutos científicos acabaron la década con un índice de inmediatez superior al del primer año, experimentando el mayor incremento el CNR, pues el valor de este indicador en 2015 para el CNR fue un 67\% superior al de 2006. Es posible apreciar cierto paralelismo entre el crecimiento de los índices de colaboración y el índice de inmediatez.

La media de citas por publicación, el porcentaje de documentos no citados y el índice h mostrados en la Tabla 14 han sido calculados para el total de lo producido por los cinco centros de investigación entre 2006 y 2015. Cuanto más alta sea la media de citas por publicación y el índice h, y menor el porcentaje de documentos no citados, mayor será el impacto de la producción de un determinado centro.

Tabla 14. Datos complementarios de impacto de la producción de los centros CNRS, HG, MPG, CSIC y CNR: media de citas, porcentaje de documentos no citados e índice h

\begin{tabular}{|l|l|l|l|l|l|}
\hline & CNRS & HG & MPG & CSIC & CNR \\
\hline Media de cit & $16,38\left(4^{\circ}\right)$ & $20,69\left(2^{\circ}\right)$ & $27,50\left(1^{\circ}\right)$ & $20,03\left(3^{\circ}\right)$ & $15,04\left(5^{\circ}\right)$ \\
\hline$\%$ de doc no cit & $18,17 \%\left(5^{\circ}\right)$ & $13,00 \%\left(3^{\circ}\right)$ & $12,73 \%\left(2^{\circ}\right)$ & $11,97 \%\left(1^{\circ}\right)$ & $16,86 \%\left(4^{\circ}\right)$ \\
\hline Índice h & $389\left(2^{\circ}\right)$ & $266\left(4^{\circ}\right)$ & $432\left(1^{\circ}\right)$ & $308\left(3^{\circ}\right)$ & $235\left(5^{\circ}\right)$ \\
\hline
\end{tabular}


Ninguna de las cinco instituciones de investigación consigue obtener el mejor de los datos para los tres indicadores. Los resultados más sobresalientes son los del MPG: logró la media de citas más alta (sus publicaciones reciben de media 27,5 citas) y el índice h más elevado (432). Su porcentaje de documentos no citados fue el segundo mejor: $12,73 \%$.

La segunda institución con mayor impacto es el CSIC: obtuvo el porcentaje de documentos no citados más bajo (11,97\%). Logró también el tercer mejor dato en cuanto a media de citas por publicación $(20,03)$ e índice h (308).

Las valoraciones más bajas se las llevaron el CNRS y el CNR. El centro francés al menos consiguió un índice h de 389, el segundo más alto. El CNR sólo se libró de la quinta posición en el porcentaje de documentos no citados, que fue de $16,86 \%$, un $1,31 \%$ inferior al del CNRS, el más negativo de todos.

\section{Conclusiones}

En este estudio, se ha analizado la producción científica del Consejo Superior de Investigaciones Científicas (CSIC) reflejada en la base de datos Scopus entre los años 2006 y 2015 y se ha comparado con la de cuatro instituciones europeas similares: CNRS, HG, MPG y CNR. Entre las limitaciones del análisis realizado, cabe mencionar que los datos se recabaron de una sola base de datos, Scopus, con los sesgos de cobertura y posibilidades de error que la caracterizan y que se explicaron en la introducción y en la metodología del artículo. Cabe añadir además que algunos de los datos que se han manejado en este trabajo no son exactos, sino aproximados como, por ejemplo, los relativos a la plantilla y al presupuesto de los centros estudiados que se recabaron de la web.

La principal aportación del estudio frente a trabajos previos similares ha sido la de comparar el CSIC con instituciones parecidas a nivel europeo, es decir, centros de investigación nacionales, en lugar de compararla con universidades y hospitales nacionales, cuya actividad principal no suele ser la investigación. Este nuevo enfoque ha permitido poner de manifiesto características diferenciales de los cinco centros, apuntando a sus peculiaridades más que evaluando su rendimiento investigador en términos de éxito o fracaso relativo. En resumen, el estudio ha mostrado el CNRS como el centro más productivo, el HG como el más especializado, el MPG como el que genera mayor impacto, el CNR como el menos colaborador, y el CSIC como el que establece la mejor relación presupuestoproducción-impacto. Se puede afirmar que la producción científica más visible y que más impacto origina es la del MPG, pues cuenta con el índice h más elevado (superior a 400) y con la media de citas por artículo más alta (superior a 27). El segundo centro con mayor impacto es el CSIC, que consiguió el mejor porcentaje de documentos no citados (cerca del 12\%) y el tercer índice h más elevado (superior a 300). También obtuvo el tercer mejor resultado en relación a la media de citas por publicación, que es algo superior a 20. En impacto, el CNRS y el CNR obtuvieron los resultados más bajos, indicando que el impacto y/o la visibilidad de las publicaciones de una entidad científica no siempre está relacionado con el 
tamaño de su producción, pues centros con una producción y una plantilla tan desigual como el CNRS y el CNR han alcanzado en este apartado valores similares. Finalmente, los centros alemanes, y especialmente el HG, destacan por llevar a cabo investigaciones especialmente innovadoras, en las que se exploran campos nunca tratados, y que requieren de una inversión económica muy fuerte. Esto podría explicar por qué, pese a contar con los presupuestos más altos, no son los centros más productivos.

El CSIC es la tercera institución científica más productiva de entre las cinco analizadas. El centro español publica casi el doble de trabajos que el centro menos productivo (el HG), pero menos de la mitad que el más fructífero (el CNRS). Sin embargo, cuenta con un presupuesto anual cuatro veces inferior al del centro francés (Tabla 1). Atendiendo a factores económicos, el CSIC también destacaría frente al MPG, pues aunque la producción del centro alemán es un 30\% superior a la del CSIC, debe tenerse en cuenta que el presupuesto que el primero ha recibido en 2016 es más de dos veces superior al del segundo. La producción científica de la década es resultado de la financiación de años anteriores, mientras que los datos aportados aquí se refieren al año 2016, según la información publicada por cada institución en su sede web. Con todas estas limitaciones, parece que el CSIC, de entre los cinco centros europeos, es el que presenta la mejor relación presupuestoproducción-impacto. Sin duda alguna, un tema interesante de trabajo para el futuro sería explicar la razón de este resultado.

La comparación del CSIC con instituciones similares en lugar que con universidades y hospitales nacionales como en estudios previos (González-Albo et al., 2012; Bordons et al., 2016), subrayando las peculiaridades de los diferentes centros, pone de manifiesto la necesidad de conocer otras facetas de la actividad investigadora y del impacto científico que puedan explicar las diferencias en características y rendimiento entre un centro de investigación y otro, como las políticas científicas o la cultura institucional. Se trata de una línea de investigación que merece la pena perseguir para visibilizar factores extrínsecos a la actividad puramente investigadora en la definición y consecución del impacto científico. La literatura está estudiando diferentes dimensiones del impacto científico, siendo de particular interés en la actualidad lo que se suele denominar el impacto social de la actividad investigadora (Bornmann, 2013). Sin embargo, la existencia de las diferencias detectadas en el presente estudio entre los cinco centros de investigación, en términos de impacto, producción, especialización o internacionalización, apunta a la necesidad de conocer también la influencia de factores políticos, sociales o de gestión en la producción científica y el impacto. De alguna manera, este análisis bibliométrico reitera las limitaciones de un enfoque puramente bibliométrico ya debatidas en cierta literatura (Waltman, 2016; MacRoberts y MacRoberts, 2018), porque, aunque se detectan diferencias entre los centros, los datos recopilados rara vez permiten ahondar en las posibles razones de las mismas.

En el presente trabajo, solo en algunos casos es posible relacionar diferentes indicadores bibliométricos para explicar ciertos fenómenos. Por ejemplo, el MPG y el CNR obtuvieron respectivamente el índice de inmediatez promedio más elevado y el más bajo. Es probable que la escasa colaboración internacional del CNR 
explique la relativa lentitud con la que se citan sus trabajos, pues la colaboración crea redes a través de las cuales la información científica puede difundirse más rápidamente que los canales tradicionales de la revista científica. En el otro extremo, el caso exitoso en términos de colaboración e inmediatez del MPG confirmaría esta posible relación. Aunque en este caso puedan verse las conexiones que existen entre diferentes indicadores bibliométricos, es importante concluir reiterando la necesidad de realizar análisis institucionales más exhaustivos que permitan aislar variables sociales, políticas, u organizativas influyentes en la actividad investigadora.

\section{Referencias bibliográficas}

Aksnes, D. W.; Taxt, R. E. (2004). Peer reviews and bibliometric indicators: A comparative study at a Norwegian University. Research Evaluation, vol. 13 (1), 33-41.

Albert, A.; Granadino, B.; Plaza, L. M. (2007). Scientific and technological performance evaluation of the Spanish Council for Scientific Research (CSIC) in the field of Biotechnology. Scientometrics, vol. 70 (1), 41-51.

Andrés, A. (2009). Measuring academic research. How to undertake a bibliometric study. Chandos Publishing; Cambridge.

Arguimbau-Vivó, L.; Fuentes-Pujol, E.; Gallifa-Calatayud, M. (2013). A decade of documentary research on Scientometrics in Spain: Analysis of papers from the ISOC database (2000-2009). Revista Española de Documentación Científica, vol. 36 (2).

Ayala-Gascón, M.; Aleixandre-Benavent, R.; Gandía-Balaguer, A. (2012). Scientific activity indicators of outstanding researchers: Scientometric portrait of Eduardo Primo Yúfera, former president of CSIC. Revista Española de Documentación Científica, vol. 35 (2), 209-237.

Beliakov, G.; Bustince-Sola, H.; Calvo-Sánchez, T. (2016). A Practical Guide to Averaging Functions. Springer.

Bordons, M.; Costas, R. (2005). Bibliometric indicators at the micro-level: some results in the area of natural resources at the Spanish CSIC. Research Evaluation, vol. 14 (2), 110-120.

Bordons, M.; Morillo, F.; Gómez, I.; Moreno-Solano, L.; Lorenzo, P.; Aparicio, J.; Álvarez-Bornstein, B.; González-Albo, B. (2016). La actividad científica del CSIC a traves de indicadores bibliométricos (Web of Science, 2011-2015). CSIC: Consejo Superior de Investigaciones Científicas; Madrid.

Bornmann, L. (2013). What is societal impact of research and how can it be assessed? A literature survey. Journal of the American Society of Information Science and Technology, 64(2), pp. 217-233.

Bosman, J.; van Mourik, I.; Rasch, M.; Sieverts, E.; Verhoeff, H. (2006). Scopus Reviewed and Compared: The coverage and Functionality of the Citation Database Scopus, Including Comparisons with Web of Science and Google Scholar. Utrecht University Library; Utrecht.

CNR. (2016). Relazione sulla Performance 2015. Consiglio Nazionale delle Ricerche. $<$ https://www.cnr.it/sites/default/files/public/media/amministrazione_trasparente/perfor mance/Relazione_Performance_2015.pdf>. [Consulta: 12/08/2017]

CNR. (2017). Il CNR in numeri. Consiglio Nazionale delle Ricerche. $<$ https://www.cnr.it/it/cnr-in-numeri>. [Consulta: 10/08/2017] 
CNRS. (2017a). 2016, une année avec le CNRS. Centre National de la Recherche Scientifique. <http://www.cnrs.fr/fr/pdf/RA2016/RA_CNRS2016_complet_BD.pdf>. [Consulta: 04/08/2017]

CNRS. (2017b). Présentation. Centre National de la Recherche Scientifique. $<$ http://www.cnrs.fr/fr/organisme/presentation.htm>. [Consulta: 02/08/2017]

Cortés Vargas, D. (2007). Medir la producción científica de los investigadores universitarios: la bibliometría y sus límites. Revista de la Educación Superior, vol. XXXVI (142), 43-65.

Costas, R., Bordons, M. Do age and professional rank influence the order of authorship in scientific publications? Some evidence from a micro-level perspective (2011) Scientometrics, vol. 88 (1), pp. 145-161.

Costas, R.; van Leeuwen, T. N.; Bordons, M. (2010). A bibliometric classificatory approach for the study and assessment of research performance at the individual level: The effects of age on productivity and impact. Journal of the American Society for Information Science and Technology, vol. 61 (8), 1564-1581.

CSIC. (2017a). Memoria 2016. Consejo Superior de Investigaciones Científicas. <http://www.csic.es/web/guest/memorias-digitales>. [Consulta: 10/08/2017]

CSIC. (2017b). Presentación. Consejo Superior de Investigaciones Científicas. <http://www.csic.es/presentacion>. [Consulta: 02/08/2017]

De Filippo, D.; Morillo, F.; Fernández, M. T. (2008). Indicators of scientific collaboration between CSIC and Latin America through international databases. Revista Española de Documentación Científica, vol. 31 (1), 66-84.

Franceschini, F., Maisano, D., \& Mastrogiacomo, L. (2016). The museum of errors/horrors in Scopus. Journal of Informetrics, 10(1), 174-182.

Elsevier. (2016). Scopus Content Coverage Guide. www.elsevier.com/_data/assets/ pdf_file/0007/69451/scopus_content_coverage_guide.pdf. [Consulta: 14/06/2017]

García, J. A.; Rodríguez-Sánchez, R.; Fernández-Valdivia, J. (2011). Ranking of the subject areas of Scopus. Journal of the Association for Information Science and Technology, vol. 62 (10), 2013-2023.

González-Albo, B.; Moreno, L.; Morillo, F.; Bordons, M. (2012). Indicadores bibliométricos para el análisis de la actividad de una institución multidisciplinar: el CSIC. Revista Española de Documentación Científica, vol. 35 (1), 9-38.

Hamilton, D. P. (1990). Publishing by-and for?-the Numbers. Science, vol. 250, 1331-1332.

HG. (2016). Annual report 2016. Helmholtz-Gemeinschaft. www.helmholtz.de/fileadmin/ user_upload/04_mediathek/2016_Geschaeftsbericht/161201_GB2016_ENGLISH_web_ A-1b.pdf. [Consulta: 10/08/2017]

HG. (2017). About us. Helmholtz Association. <https://www.helmholtz.de/en/about_us/>. [Consulta: 07/08/2017]

Hirsch, J. E. (2005). An index to quantify an individual's scientific research output. PNAS: Proceedings of the National Academy of Sciences of the United States of America, vol. 102 (46), 16569-16572.

Krauskopf, E. (2017). Call for caution in the use of bibliometric data. Journal of the Association for Information Science and Technology, vol. 68(8), 2029-2032.

López-Muñoz, F.; Marín, F.; Boya-Vegue, J. (1996). Evaluación bibliométrica de la producción científica española en Neurociencia. Análisis de las publicaciones de difusión internacional durante el período de 1984-1993. Revista de Neurología, vol. 24 (128), 417-426. 
MacRoberts, B. R.; MacRoberts, M. H. (2010). Problems of citation analysis: A study of uncited and seldom-cited influences. Journal of the American Society for Information Science and Technology, vol. 61 (1), 1-13.

MacRoberts, M. H., \& MacRoberts, B. R. (2018). The mismeasure of science: Citation analysis. Journal of the Association for Information Science and Technology, vol. 69(3), 474-482.

Mauleón, E.; Bordons, M.; Oppenheim, C. (2008). The effect of gender on research staff success in life sciences in the Spanish National Research Council. Research Evaluation, vol. 17 (3), 213-225.

Mesnard, L., de (2012). On some flaws of university rankings: the example of the SCImago report. The Journal of Socio-Economics, vol. 41(5), 495-499.

Mongeon, P.; Paul-Hus, A. (2016). The journal coverage of Web of Science and Scopus: a comparative analysis. Scientometrics, vol. 106 (1), 213-228.

MPG. (2017a). Annual Report 2016. Max-Planck-Gesellschaft. $<$ https://www.mpg.de/11359014/annual-report-2016.pdf >. [Consulta: 10/08/2017]

MPG. (2017b). A portrait of the Max Planck Society. Max-Planck-Gesellschaft. $<$ https://www.mpg.de/short-portrait>. [Consulta: 07/08/2017]

Orduña-Malea, E.; Martín-Martín, A.; Ayllón, J. M.; Delgado López-Cózar, E. (2016). La revolución Google Scholar: Destapando la caja de Pandora académica. Unión de Editoriales Universitarias Españolas; Granada.

Ortega, J. L. (2011). Collaboration patterns in patent networks and their relationship with the transfer of technology: the case study of the CSIC patents. Scientometrics, vol. 87 (3), 657-666.

Ortega, J. L. (2017). Toward a homogenization of academic social sites: A longitudinal study of profiles in Academia. edu, Google Scholar Citations and ResearchGate. Online Information Review, vol. 41 (6), 812-825.

Rivera-Hernández, M. P. (2015). Análisis de la producción científica en revistas de biblioteconomía y documentación en México (2009-2013): aplicación del método de análisis cualitativo de citas. (Tesis doctoral), Universidad Complutense de Madrid; Madrid.

Romo-Fernández, L. M.; Guerrero-Bote, V. P.; de Moya-Anegón, F. (2013). Analysis of the Spanish scientific production in renewable energy, Sustainability and the Environment (Scopus, 2003-2009) in the global context. Investigación Bibliotecológica, vol. 27 (60), 125-151.

Sánchez, M. (1999). Análisis comparativo de la producción científica de la Unión Europea, España y el Consejo Superior de Investigaciones Científicas. CSIC: Consejo Superior de Investigaciones Científicas; Madrid.

SCImago Lab. (2015). Western European Government Institutions Ranking. SCImago Institutions Rankings. <http://www.scimagoir.com/rankings.php?sector=Government\& country=Western\%20Europe\&year=2009>. [Consulta: 01/03/2017]

Scopus. (2006). Scopus Content Coverage. <http://www.library.ait.ac.th/download/ Scopus_content_coverage.pdf $>$. [Consulta: 19/06/2017]

Seglen, P. O. (1997). Why the impact factor of journals should not be used for evaluating research. BMJ: British Medical Journal, vol. 314, 498-502.

Spinak, E. (1998). Indicadores cienciométricos. Ciência da informação, 27(2), 141-148.

Thelwall, M., \& Kousha, K. (2015). Web indicators for research evaluation. Part 1: Citations and links to academic articles from the web. El profesional de la Información, vol. 24(5), 587-606. 
Torra, V.; Narukawa, Y. (2008). The h-Index and the Number of Citations. Two Fuzzy Integrals. IEEE Transactions on Fuzzy Systems, vol. 16 (3), 795-797.

Valderrama-Zurián, J. C., Aguilar-Moya, R., Melero-Fuentes, D., \& Aleixandre-Benavent, R. (2015). A systematic analysis of duplicate records in Scopus. Journal of Informetrics, vol. 9 (3), 570-576.

van Raan, A. F. (2004). Measuring science. En Handbook of Quantitative Science and Technology Research. Kluwer Academic Publishers, p. 19-50; Dordrecht.

van Raan, A. F. (2005). "Fatal attraction: Conceptual and methodological problems in the ranking of universities by bibliometric methods”. Scientometrics, 62(1), 133-143.

Velasco, B.; Eiros, J. M.; Pinilla, J. M.; San Román, J. A. (2012). La utilización de indicadores bibliométricos para evaluar la actividad investigadora. Aula Abierta, vol. 40 (2), 75-84.

Vieira, E. S., \& Gomes, J. A. (2009). A comparison of Scopus and Web of Science for a typical university. Scientometrics, vol. 81(2), 587.

Waltman, L. (2016). A review of the literature on citation impact indicators. Journal of Informetrics, vol. 10(2), 365-391. 